\title{
Video assisted thoracoscopic surgery for spinal conditions
}

\author{
Gabriel K. P. Liu, Wong Hee Kit \\ Division of Spine Surgery, Department of Orthopeadic Surgery, National University Hospital, 5 Lower Kent Ridge Road, Main Building I, \\ Level 3, Singapore - 119074
}

\begin{abstract}
Video-assisted thoracoscopic surgery (VATS) has become an alternative treatment tool for a variety of spinal conditions in the last two decades. This endoscopic or 'keyhole' approach minimizes the chest wall morbidity related to the traditional thoracotomy. The current indications for VATS are the same as in any open anterior spinal surgery. This article reviews the outcomes of VATS treatments in thoracic disc diseases, fractures, tumors and vertebral osteomyelitis. In addition, we have described our 'learning curve ' and surgical techniques using video-assisted thoracoscopic spinal releases and instrumentation in the treatment of 50 patients with adolescent idiopathic scoliosis.
\end{abstract}

Key words: Video-assisted thoracoscopic surgery, spinal surgery

\section{Introduction}

Recent technological advancement in endoscopic surgery has revolutionalised all traditional surgical approaches in the past two decades. ${ }^{[3,16,20]}$ Video-assisted thoracoscopic surgery (VATS) has become a keyhole to the minimally invasive or access surgical approaches to the thoracic spine. ${ }^{[7,18,20,40]}$

The first clinical report of thoracoscopic surgery was described in 1910 after Jacobaeus used a thoracoscope to diagnose and lyse the tuberculosis lung adhesion. ${ }^{[16]}$ With the discovery of streptomycin in 1945 for tuberculosis treatment, there was a decreased in clinical application of thoracoscopy for such condition. ${ }^{[14]}$ It was not until Lewis in 1991 had repopularised the use of VATS for pulmonary disease treatment and Mack in 1993 reported the application of VATS for spine disease, the enthusiasm in using VATS for spine condition has exploded.

VATS involved the use a fiberoptic camera and a light source for visualization and magnification through small percutaneous portals. The aim is to target the pathology with minimal damage to the surrounding non-pathological tissue. It offers a direct illumination and up to 15 times magnification of the subject. By varying the placement of the thoracoscope, scope angle and camera trajectory; VATS allow a clear visualization of the extreme ends of the spine from $\mathrm{T} 1$ to $\mathrm{T} 12$ which would otherwise be difficult in the traditional thoracotomy approach.

In addition VATS retained the advantages of anterior spinal surgery and gave a comparable result of spinal deformity correction to that of the open approaches ${ }^{[4,18,25,26,27,40]}$ (Table 1). VATS had significantly reduced the chest wall morbidity related to the traditional thoracotomy. ${ }^{[25]}$ These included a reduction of the post-operative incisional pain and intercostal neuralgia. ${ }^{[26,29]}$ There is a less reduction of forced vital capacity ${ }^{[25]}$ and a trend towards greater return of shoulder girdle strength and range of motion at 6 weeks after operation. ${ }^{[25,26]}$ VATS allows a greater access to more vertebral levels through small incisions. It resulted in a less intra-operative bleeding, shorter hospital stay, earlier return to work ${ }^{[1,20]}$ and smaller cosmetically acceptable scars. ${ }^{[16,27,40]}$

As VATS technology developed and surgical experiences grew, the indications for VATS should be the same as when an open thoracotomy procedure is considered (18). The use of VATS in spine surgery included the treatment of thoracic prolapsed disc diseases, ${ }^{[3,32]}$ vertebral osteomyelitis, ${ }^{[10,13,20,23]}$ corpectomy for selected tumor, ${ }^{[8,12,15,21,31]}$ fracture management, ${ }^{[11]}$ vertebral

Table 1: Advantages and disadvantages of VATS versus traditional thoracotomy approach

\begin{tabular}{|c|c|}
\hline Advantages & Disadvantages \\
\hline Better spinal visualization & Steep learning curve \\
\hline Decreased incisional pain & Required double lumen intubation \\
\hline Decreased blood loss & $\begin{array}{l}\text { Limitations in dealing with intra- } \\
\text { operative complications }\end{array}$ \\
\hline Decreased ICU \& hospital stay & Lack of stereoscopic appreciation \\
\hline $\begin{array}{l}\text { Decreased shoulder } \\
\text { dysfunction }\end{array}$ & d specialized equipment \\
\hline $\begin{array}{l}\text { Decreased pulmonary } \\
\text { dysfunction }\end{array}$ & Required fluoroscopy \\
\hline Cosmetically acceptable scars & Costly \\
\hline
\end{tabular}


interbody fusion,$^{[3]}$ tissue biopsy ${ }^{[10,16]}$ anterior spinal release and fusion without ${ }^{[1,2,7,14,18,24-26,36,38]}$ or with instrumentation (VAT-I) for spinal deformity correction. ${ }^{[27,30,40]}$

The absolute contraindication for VATS includes ones inability to tolerate single-lung ventilation; $\mathrm{FEV}_{1}$ less than $50 \%{ }^{[16]}$; dense pleural adhesion; respiratory insufficiency; empyema and failed prior thoracotomy surgery.

Although VATS are used in many spine conditions, it is probably most beneficial in the treatment of scoliotic deformity. This is because in scoliosis, there is a need to access multiple vertebrae and intervertebral discs, from the upper to the lower thoracic spine in contrast to conditions like thoracic disc prolapse or infection where the pathology is localized to one or two segmental levels, and where an alternative open procedure like mini-open thoracotomy could be easily used. In scoliosis, multiple portals in the lateral chest wall provide unrestricted and 'in-line' access to the thoracic vertebrae and intervertebral spaces from T4 to L1 (Figure 1).

An ideal surgical candidate for video-assisted thoracoscopic spinal instrumentation (VAT-I) in scoliosis correction would be a right side thoracic, adolescent idiopathic scoliotic (AIS) curve of King 3 or Lenke type $1^{[17]}$ (Table 2 ). The magnitude of the structural curve should be less than $80^{\circ}$. Thoracic kyphosis should be less than $40^{\circ}$ and the patient weight ranged between 30 to $70 \mathrm{~kg}$. Extended indications for VAT-I in King type 2, Lenke type 3 and type 5 curve may be considered.

Before embarking on VATS, one must be familiar with the surgical anatomy, anesthetic requirement, patient positioning and the endoscopic techniques to ensure optimal surgical outcome.

\section{Surgical anatomy and its clinical relevance}

The majority of VATS approach for spinal pathology is from the right side where there is a greater working spinal surface area lateral to the azygos vein than that to the aorta. ${ }^{[9]}$ Below T-9, a left-sided approach is made possible that the aorta has moved away from the left posterolateral aspect of the spine to an anterior position as it passes through the diaphragm.

The intervertebral disc is identified as the undulating prominence along the spinal column whereas the vertebra is situated at the bottom between these prominences. The disc is therefore regarded as the analogue of a mountain and the vertebral bodies as the valleys between these mountains. The single most important anatomical landmark within the thoracic cavity was the position of the rib head. The rib numbers correspond to the lower vertebral body and articulate with the demi facets above and below the disc space (e.g. the 8 ribs comes off the T7-8 disc space). Thus the rib head serves as a guild to the disc space, a landmark for the vertebral body screw insertion and an imaginary boundary to

Table 2: Indications for VAT-I in AIS

Curve pattern: King 3, Lenke 1

Cobb angle $<80^{\circ}$

Apical Rotation < 3 (Nash and Moe)

Patient weight between $30-70 \mathrm{~kg}$ alert surgeon not to work posteriorly beyond the rib head into the spinal canal.

The ligation of the segmental vessels at the waist of the vertebral body had been a controversial technical issue. (Figure 2) Although Winter $^{[39]}$ demonstrated no neurological deficit noted in ligating more than 6,000 vessels in 1,197 patients, other authors via animal models and clinical review caution one on the potential reduction of cord perfusion in scoliotic patient with neurological deficit, severe kyphosis, congenital deformity and revision surgery. ${ }^{[28,35]}$ It is our practice to preserve these vessels when doing discectomies alone and without instrumentation and to divide and retract the vessels to expose the vertebral body for screw insertion. The branches of the symphatic chain coursing anteriorly across the vertebral bodies are often divided during pleura dissection along the spinal column. It might lead to transient vasodilatation of the ipsilateral leg, which the patient often reports as an 'apparent' cold sensation on the non-operated contralateral leg. The effect on ipsilateral transection of the splanic nerves is less defined. We feel it might possibly result in the reduction of gastrointestinal motility and a nauseous sensation felt by the patient in the early postoperative period.

The spinal levels may be determined during thoracoscopic surgery by locating the superior intercostal vein emptying into the superior azygos vein at T3-4 interspace and by identifying the diaphragmatic insertion at the vertebrae at the caudal aspect. The median accurate ligament is formed by the unification of the left and right crura, which strapped the aorta onto T12 vertebra body anteriorly. The individual crus originated laterally at the upper body of L1. These features facilitate the location of the diaphragm at T12 vertebra and T12-L1 disc space. Ultimately the presiding factor for any spinal level identification is by taking an intra-operative radiograph to localize an intervertebral disc marked by a Steinman pin.

\section{Anesthesia requirements}

A key component to ensure a smooth running of the thoracoscopic procedure is the use of a selective double lumen endotracheal intubation method which allows a full collapse of the lung on the operative side during anesthesia in a lateral decubitus patient position. Alternatively, a bronchial blocker may be employed in a patient who weighs less than $45 \mathrm{~kg}$ in whom the smallest endotracheal tube may not fit the bronchial system. ${ }^{(24)}$ However, the reliability of a bronchial blocker to achieve a full collapse of the lung is often less predictable.

The patient is turned to the left lateral position, with the right side of the chest upwards. This position is maintained by flexion of the downside hip and knee, and secured by using surgical tapes. An axillary role is positioned to prevent pressure on the dependent shoulder. It is important that no metal supports block imaging of the thoracic spine during the course of surgery.

Newton et al has successfully demonstrated the safety of anterior thoracoscopic spinal release and fusion without instrumentation in the patient less than $30 \mathrm{~kg}$ of weight. He pointed out that in these small children, greater blood loss per kg body weight and 


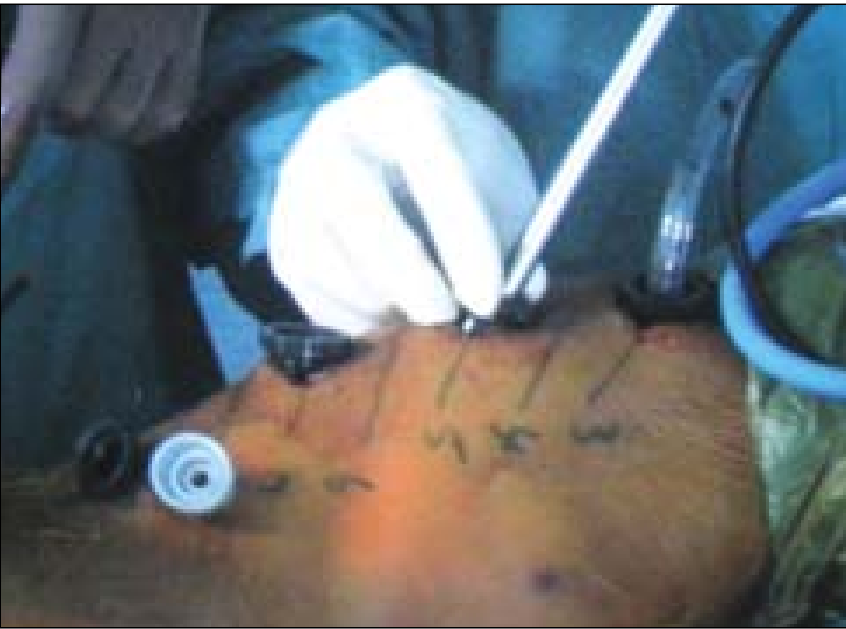

Figure 1: Shows an all posterolateral VATS approach

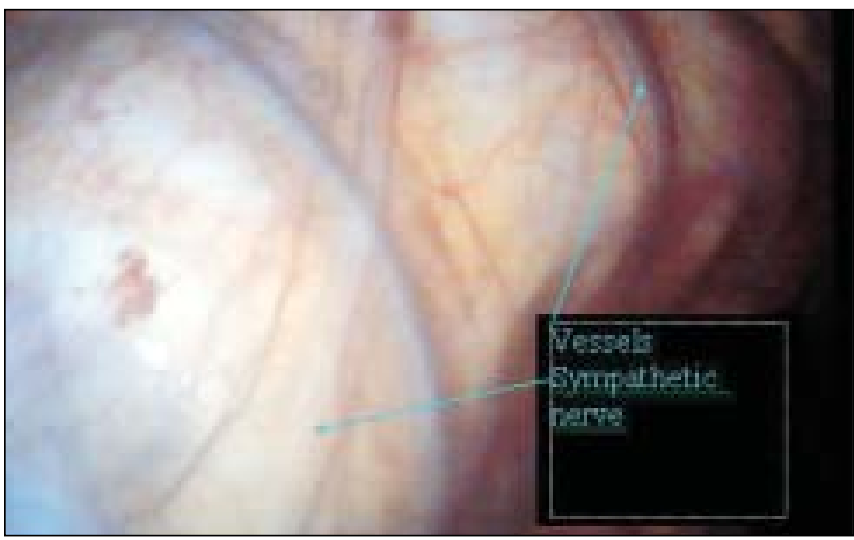

Figure 2: Shows the segmental vessel and the sympathetic nerve.

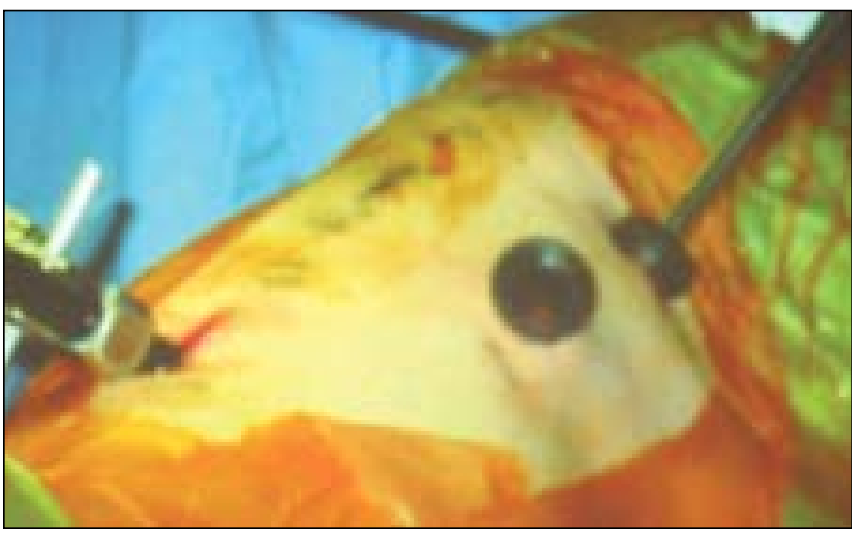

Figure 3: Shows a combined anterolateral approach for discectomies and posterolateral approach for spinal instrumentation

specialized methods of intubation for single lung ventilation and a longer ICU stay might be required. A relative contraindication for VATS in-patient of less than $20 \mathrm{~kg}$ body weight should be considered.

Recently the execution of an anterior thoracoscopic soft tissue release on patient in a prone position had been examined. ${ }^{[14,19,36]}$ The advantage includes a reduction in patient reposition time and a two-surgery technique for a concomitant anterior spinal

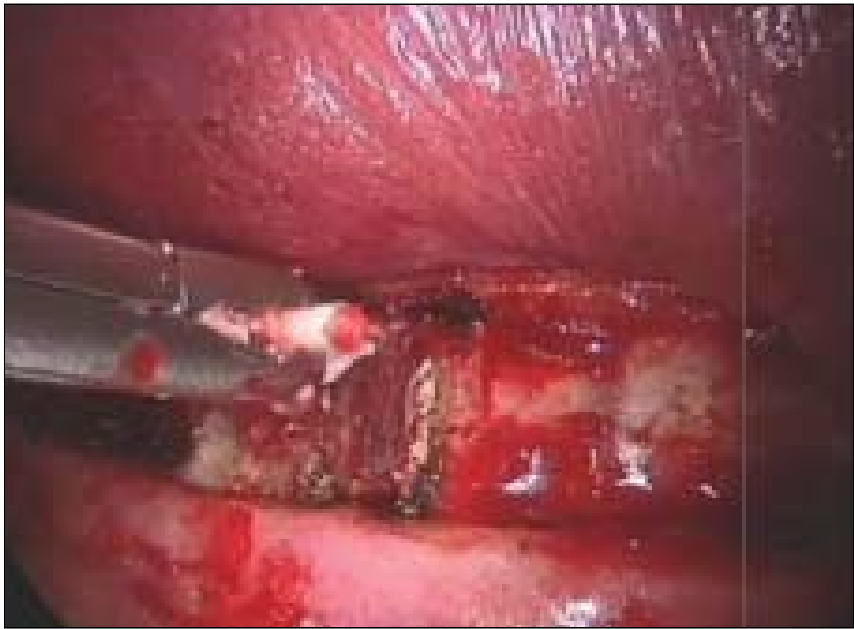

Figure 4: Shows thoracoscopic discectomy for anterior fusion.
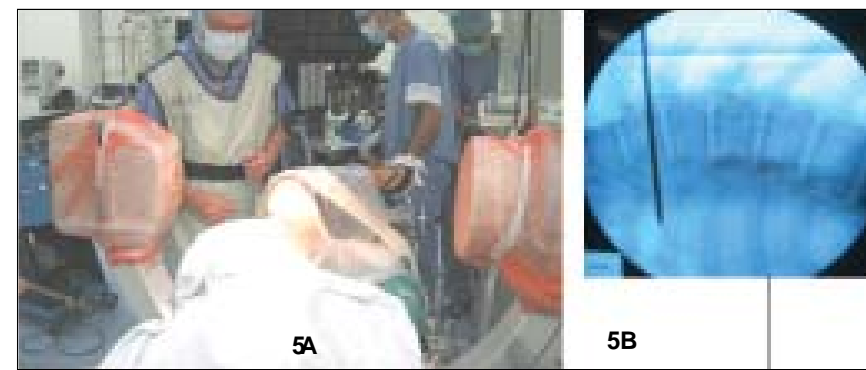

Figure 5a and 5b: Show the positions of the $\mathrm{C}-\mathrm{arm}$, and the corresponding fluoroscopic spinal images.
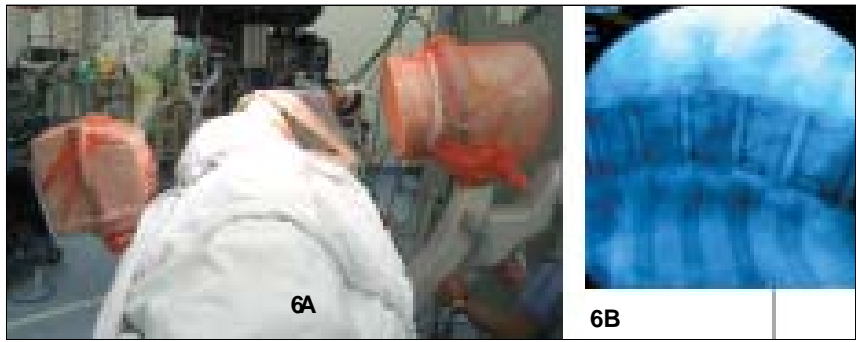

Figure 6a and 6b: Show the positions of the C-arm, and the corresponding fluoroscopic spinal images. The degree of vertebra rotation is accessed. The C-arm of the fluoroscope is rotated from its horizontal position (Figure 5a) until an image of a Neutral Vertebra of Moe is obtained on the AP spine fluoroscopic view (Figure 6a and b). Note the asymmetric pedicles of the thoracic spinal image in figure $5 \mathrm{~b}$ compared to the 'squared' symmetric pedicles of the true AP thoracic spinal image in figure $6 \mathrm{~b}$. The angle from which the $\mathrm{C}$-arm is being rotated away from the horizontal plane to achieve the true AP image represents the degree of vertebral rotation at that spinal level.

release and a posterior spinal instrumentation and fusion in a scoliotic patient had been encouraging. In addition, the lung falls anteriorly away from the spine and obviates the need for lung retraction and the pre-requisite of a double lumen intubation in conventional VATS. ${ }^{[14,36]}$ This result in less total oxygen requirement and an earlier hospital discharge. ${ }^{[36]}$ Other advantages included a gravitational reduction in kyphotic deformities after anterior release and the ease of performing an extended 
costotransversectomy should the need arise. However it is unclear whether bone graft placed in this prone patient position will remain in the disc space and the safety for deformity correction and instrumentation are yet to be validated. ${ }^{[18]}$

\section{Principle of endoscopic approaches}

Currently two strategies for endoscopic portal placements have emerged. In an anterolateral approach the surgeon stand on the patient ventral side to face the patient, by triangulation, more spinal levels can be approached form each portal especially in the presence of a large thoracic kyphosis. A more global visualization of the entire spine and a possible glimpsed to the anterolateral surface of the contralateral spinal surface may be seen. This facilitates a thorough discectomy especially at the anterior, posterolateral corners of the disc and the removal of the annulus situated deep in the concave side of the curve that would otherwise be difficult in an all posterolateral approach.

Anterolateral approach also allows the ease of a surgical plane dissection between the azygos vein and the vertebrae. The spine could be fenced by temporary gauze placement in this plane. This would maintain a clear visual to the spine by mopping up the unavoidable bleeding from the vertebral end plates and add extra protection to the anterior spinal structures during spinal release. ${ }^{[27]}$

In a combined anterolateral and posterolateral approach, the portals are first placed along the anterior axillary line for spinal release and fusion; ${ }^{[7,26,38]}$ then replaced posterolaterally for vertebral bone screw insertions ${ }^{[27]}$ (Figure 3).

A disadvantage is the potential danger of working with instrument from an anterior to posterior direction into the spinal canal. The needed of additional posterolateral portal for instrumentation in scoliotic deformity correction may add surgical morbidity and the number of scars otherwise be avoided in an all posterolateral endoscopic approach. The position of the anterolateral portals may also be less cosmetically acceptable because of their relatively anterior position and the proximity to the right breast, particularly if the healing scar becomes hypertrophic.

In an all posterolateral approach, all access portals are placed between the mid and posterior axillary lines ${ }^{[30,40]}$ (Figure 1).

The surgeon stands to face the back of the patient. This approach allows a natural transition of the VATS surgeon in training from a usual thoracotomy approach to a VATS approach. In addition both discectomy with fusion and instrumentation could be performed via these posterolateral portals without the need of additional anterolateral portals. Another advantage is the inherent safety of this approach, which force the surgeon to work away from the spinal canal in a posterior to anterior direction during spinal instrumentation.

The disadvantages related to an all posterolateral approach are the increased technical difficulties in performing a thorough discectomy particularly in clearing the deep annulus fibers at posterolateral corner of the disc. A lack of protection to the anterior vascular structure during the anterior longitudinal ligament (ALL) release may be a concern. However, it had been demonstrated that a comparable surgical deformity correction could be achieved via an all posterolateral VAT-I approach to that of the conventional posterior instrumentation and fusion in patient with AIS. ${ }^{(40)}$ A potential complication is an injury to the long thoracic nerve resulted in a medical scapular winging from the first and possible second cephalic posterior portal insertion between the 3rd and 5 th ribs. Detailed knowledge of the nerve anatomy would help to minimize the risk of such injury during an all posterolateral VAT-I approach.

\section{Surgical technique: Discectomy}

A successful intervertebral fusion and deformity correction requires a thorough discectomy ${ }^{(16)}$ and end plate clearance. The parietal pleura on the spinal column are incised longitudinally along the peak of the disc where it is most avascular. This is best achieved by using a harmonic scalpel (Ultracision LCS, Ethicon Endosurgery Inc. Piscatawy, NJ). The harmonic scalpel is used like a paintbrush, and the pleura are gradually brushed away to expose the anterior longitudinal ligament (ALL) anteriorly and the rib head posteriorly.

The same paintbrush technique is applied to cauterize the intervertebral segmental vessels. Care is required not to cauterize these vessels in a single firm stroke for that would result in a semicauterized vessel, which would bleed profusely. The vessel should be cauterized and brushed away slowly, layer by layer until the vessel turned grey then browned before it is severed. There is often bleeding from the nutrient vessel that runs from the segmental vessel perpendicularly into mid posterior portion of the vertebrae; this bleeding is often stop by plugging the bleeding spot with bone wax applied on a thoracoscopic peanut. It should be emphasized that clear surgical field with minimal bleeding facilitates the thoracoscopic procedure.

Once the intervertebral disc is exposed beneath the pleura, the disc borders are outlined by cautery; the annulus is then incised by a long handled no. 15 scalpel blade. A pituitary rongeur is used to remove the annulus disc complex. The cartilaginous end plates are separated from the subchondral vertebral bone by using a sharp cut Cobb elevator; and the final clearance of the disc space is carried out by a combination of straight and angled pituitary rongeurs and cup curettes.

Partial released of ALL is often adequate ${ }^{(40)}$ and the residual ALL may assist in retaining the bone graft in the disc space. The adequacy of the intervertebral disc release can be accessed by the mobility of the disc space created by a gentle rotational movement using a Cobb elevator. The more effective is the disc release, the more the disc spaces close up on each other as surgery progresses. The posterior longitudinal ligament (PLL) is not incised during anterior spinal release and may acts as a protective barrier to the spinal cord.

Once discectomy is complete, a piece of gelfoam or surgicel is inserted into the disc space to tamponade the intradiscal bleeding before bone grafting. The average time for a single level discectomy in scoliotic deformity correction varies between 20 to 30 minutes ${ }^{[1]}$ (Figure 4). 
Discectomy time would be increased should the indication of discectomy be for neural decompression. The resection of the proximal $2 \mathrm{~cm}$ of rib head (except when the level was below T11) is required to achieve thorough clearance at the posterolateral corner of the disc. ${ }^{[3]}$ The foramenal ligaments are then cut to expose the superior edge of the pedicle. The superior part of the pedicle is resected to expose the spinal canal. The posterolateral corner of the disc or disc herniation can be traced from the superior edge of the pedicle. Adequate disc clearance can be determined by the free passage of a nerve dissector anterior to the PLL and by radiographic localization of the dissector tip to ascertain that the decompression had extended past the midline of the spinal canal. ${ }^{[3]}$

To ensure an optimal visualization needed for disc space clearance, a $30^{\circ}$ or $45^{\circ}$ angled $10 \mathrm{~mm}$ telescope is used to visualize the intervertebral disc space. In addition, a direct visual into the disc space and vertebrae is often helpful, and this can be easily achieved by looking down through the portal openings before the insertion of any instrumentation. This gives an additional threedimensional view of the spine that is unattainable on the endoscopic video image.

Surgical Techniques: Portal localization, Spinal fusion and Spinal Instrumentation for a Right sided Adolescent Idiopathic Scoliosis

\section{Portal localization}

Incisions for the thoracoscopic ports are centred over the ribs; typically the approach to instrument a curve from T5 to T12 will have incisions made over the $3^{\text {rd }}, 5^{\text {th }}, 7^{\text {th }}$, and $9^{\text {th }}$ ribs. Entry into the chest is made over the cephalad and caudal edges of each rib resulting in 8 entry portals from just 4 chest wall incisions. The $3^{\text {rd }}$ rib incision allows instrumentation of the $5^{\text {th }}$ and $6^{\text {th }}$ vertebrae; $5^{\text {th }}$ rib incision, the $7^{\text {th }}$ and $8^{\text {th }}$ vertebrae; and so on. The intervertebral discs could be reached easily with these incisions. The antero-posterior positions of the incisions vary depending on the axial rotation of the end and apical vertebrae; the placement being made under image intensifier control to avoid instrument violation of the spinal canal (Figure 5-7). Typically, the $3^{\text {rd }}$ and $9^{\text {th }}$ rib incisions are placed at the mid-axillary line while the $5^{\text {th }}$ and $7^{\text {th }}$ rib incisions are at the posterior axillary line. If the instrumentation needs to be performed from $\mathrm{T} 6$ to $\mathrm{L} 1$, the incision array is moved caudally, onto the $4^{\text {th }}, 6^{\text {th }}, 8^{\text {th }}$, and $10^{\text {th }}$ ribs. Preoperatively, these portal positions can be marked out on a chest radiograph and adjustments made for any undue sloping of the ribs. The first entry into the chest should be made at the midchest incisions and not through the lowest rib incision. This is to avoid inadvertent penetration through the diaphragm and into the abdomen as a result of the higher diaphragmatic dome on the right side.

A 2-cm skin incision is cut parallel to the rib. It is important not to have a large skin incision and thus de-stabilized the press-fit required for a stable $11.5 \mathrm{~mm}$ diameter semi-rigid portal placement through the chest wall. A 1 in 2 million adrenaline preparation is injected into the incision site to reduce the subdermal bleeding.
The soft tissue is divided along the rib by a cautery. Lung ventilation in the operative side is blocked and one-lung ventilation on the non-operative side is achieved. The partial pleura on the chest wall are incised at the superior border of the rib. A pneumothorax is created and the pulmonary parenchyma collapsed to allow the exposure of the spinal column. Gentle dissection must be employed to avoid iatrogenic pulmonary parenchyma injury during the first portal insertion, for the lung will not be fully collapsed until the pleura are cut and thus parenchyma is still residing closely beneath the rib cage during the first pleural incision. The remaining portals are inserted under direct thoracoscopic vision. In addition, a small $5.5 \mathrm{~mm}$ diameter portal is inserted anterior to the anterior axillary line at 8th intercostal space. This portal allows the insertion of a thoracoscopic peanut and provides an opening for the postoperative chest tube insertion for pleural cavity drainage.

The surgeon may stand either at the back or in front of the patient. I generally prefer standing facing the patient's back. The assistant who holds the scope stands on the same side of the surgeon to avoid disorientating mirror-image effects; and another assistant stands opposite the surgeon to help with retraction. 10 $\mathrm{mm}$ telescopes with $30^{\circ}$ or $45^{\circ}$ viewing angles are used. A single monitor is sufficient for surgical team although more monitors may provide convenient viewing for others in the operating room. The collapsed lung does not usually come in the way; there is little need for formal retraction of the lung. A small thoracoscopic peanut may be used to retract the diaphragm to provide access to T12 and with minor diaphragmatic detachment, to reach L1.

\section{Spinal fusion}

Following after discectomies, segment of the rib under the skin incisions are removed via open rib harvesting technique and rib cutter. This provides morselized autogeneous rib graft for intervertebral body fusion and a possible thoracoplasty effect. Alternatively, the rib graft can be harvested via a closed endoscopic technique ${ }^{[22]}$ or iliac crest graft could be used. ${ }^{(18)}$ The rib ends can also be sutured back together through drill holes to provide chest wall stability. ${ }^{[30]}$ The use of autogeneous bone graft for arthrodesis in VAT-I procedure is the current standard treatment of choice. ${ }^{[16]}$ High incidence of pseudoarthrosis is noted if demineralized bone matrix or allograft is to be used. ${ }^{[29]}$

\section{Vertebral bone screw insertion}

The vertebral screw entry point is located just anterior and inferior to the corresponding rib head. This often coincides with the entry point of the nutrient artery in the middle portion of the posterior $1 / 3$ of the vertebra. Irrespective of screw insertion technique used, it is important to ensure the rotational component of each vertebra in the deformity is fully appreciated. Perpendicular screw insertion into a vertebra without the allowance of vertebral rotation will result in an iatrogenic spinal canal penetration by the screw. To avoid this, an image of a Neutral Vertebra of Moe should be obtained on the $\mathrm{AP}$ view on the 


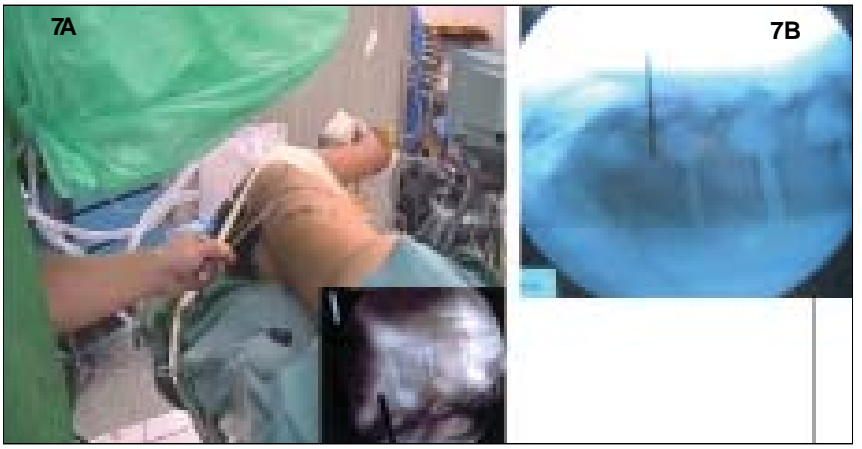

Figure $7 \mathrm{a}$ and $7 \mathrm{~b}$ : Show the $\mathrm{C}$ arm is rotated away from its vertical position to accommodate the rotational deformity of each vertebra.

The corresponding true lateral image of the vertebrae is shown.

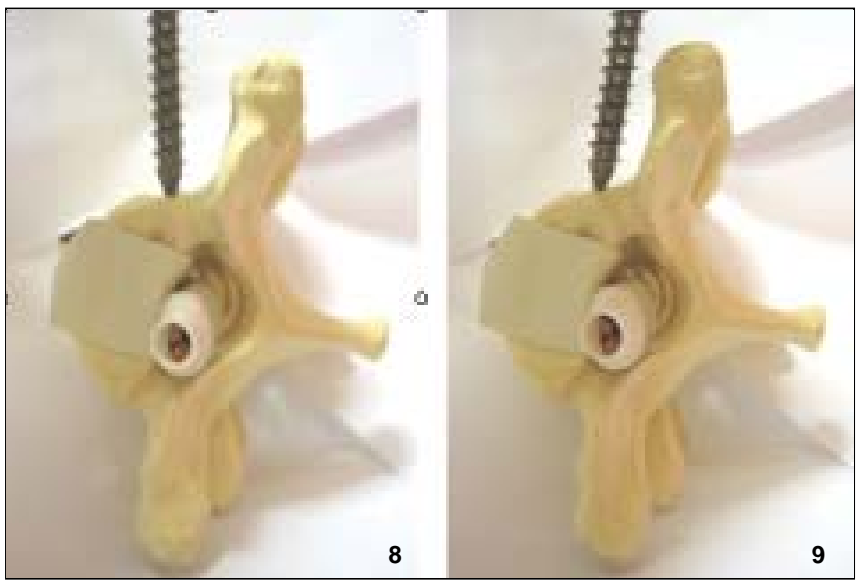

Figure 8: Shows a potential iatrogenic spinal canal penetration trajectory by the screw if the trajectory of screw insertion is not adjusted to accommodate the rotational component of each vertebra. Figure 9: Shows an adjusted screw projectory.

fluoroscope before any instrumentation procedure to be performed on that vertebra. Instrument directed into the spine should be placed perpendicular to the imaginary plane between the x-ray tube and the image intensifier on either ends of the C-arm. This would avoid iatrogenic spinal canal penetration by instruments (Figures 8 and 9).

The exact techniques of screw insertion will depend on the particular type of thoracoscopic instrumentation used.The cannulated screw system (Eclipse system, Sofamor-Danek, Memphis, TN), a guide wire is inserted under fluoroscopic control into the vertebral body (Figure 10). The guide wire maintains the trajectory of the screw following tapping of the outer cortex. It is important to ensure that the guide wire does not penetrate beyond the far cortex of the vertebrae. Inadvertent guide wire advancement may also occur during cannulated tapping and screw insertion over the wire. ${ }^{(30)}$ Guide wire advancement beyond the far cortex of the vertebra may injure the contralateral pulmonary parenchyma and vessels. ${ }^{(2,29,33)}$ Another technique is direct screw placement without prior guild wire insertion. The screw entry point at the lateral aspect of the vertebral body is prepared with a bone awl (Frontier system, De-Puy, AcroMed, Raynham, MA) under both endoscopic and fluoroscopic control (Figure 11). The screw hole is then tapped and the screw inserted.

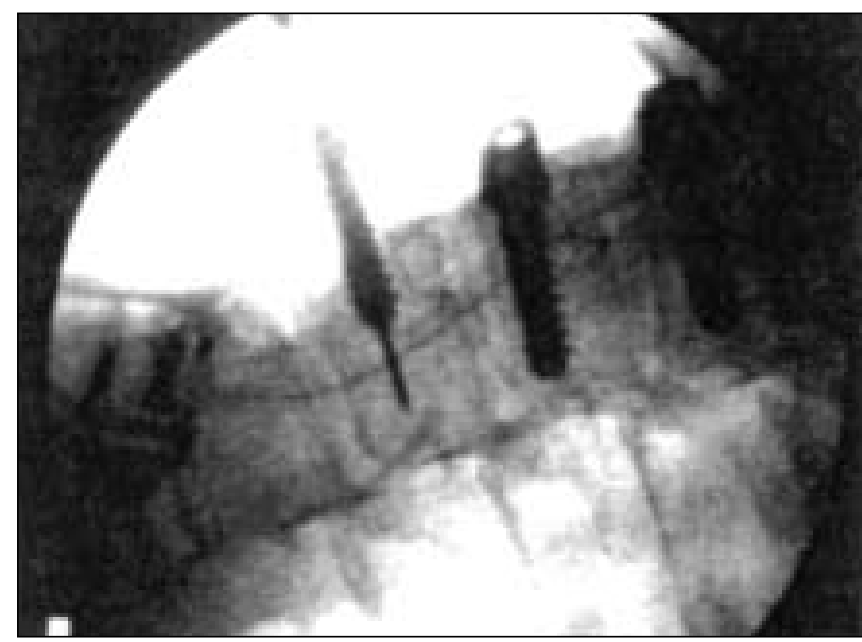

Figure 10: Shows screw insertion using a guild wire technique.

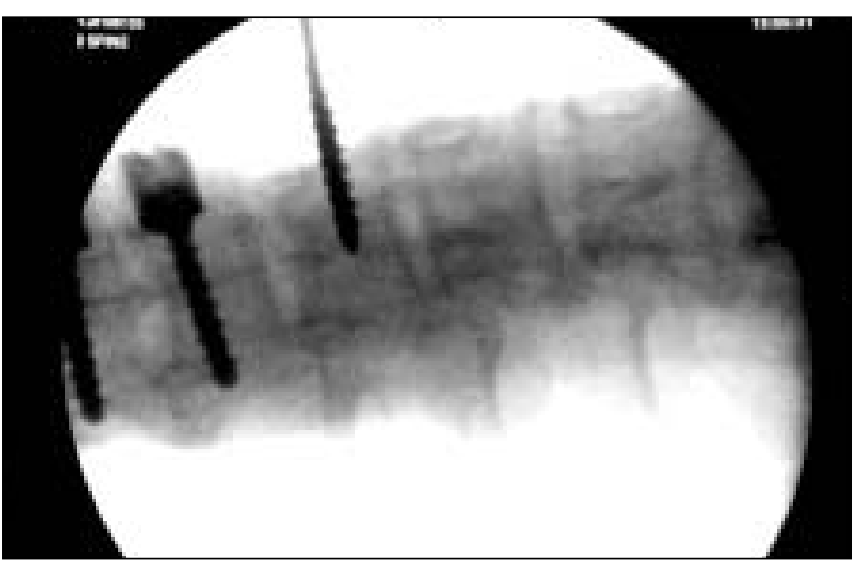

Figure 11: Shows screw insertion using an awl technique

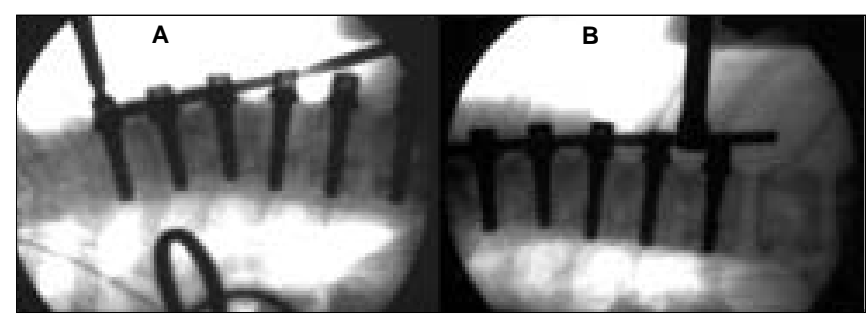

Figure 12 (a) and (b): Show a scoliosis reduction by a cantilevered method

The final screw position should be in the middle of the vertebral body and parallel to its vertebral end plates. Bicortical screw purchase is preferable. It is critical to ensure that each screw head is placed against the near cortex of each vertebra; and that the screws are in a progressive line in a cephalo-caudal direction to avoid abrupt screw offset in either height or axial alignment. Misalignment of one or more screws could lead to difficulty in engaging the rod into the screw heads. Fractures of the vertebral body could even occur during forced rod engagement. ${ }^{[27]}$ It has been suggested rib head resection at or above T6 should be performed to ensure the screw could be placed posterior enough on the vertebral body or allow for the space required for a staplescrew complex to be inserted if desired. ${ }^{[18]}$ 
Instrumentation systems that allow for small screw length increments (e.g. $2.5 \mathrm{~mm}$ per interval) are preferable to avoid the placement of excessive long screws, where the screw tip could impinge on the aorta on the contralateral side of screw insertion. ${ }^{[37]}$

In circumstances in which a placement of a L2 screw is required, a small retropertioneal incision is created in line with the more cephalic placed posterolateral portals to allow discectomy and fusion at L1/L2. Subsequent rod to screw placement is performed with the rod being passed under the diaphragm after detaching its vertebral attachment to reach the upper lumbar vertebrae. No formal detachment of the diaphragm is needed. ${ }^{[40]}$ It is advised that interbody cages or structural bone graft should be placed below $\mathrm{T} 11^{(27)}$ to avoid the induction of a kyphotic deformity in the thoracolumbar junction during scoliosis reduction.

\section{Rod engagement and scoliosis deformity reduction}

An endoscopic rod length-measuring device can be used to obtain an appropriate length of the reduction rod (Eclipse system, Sofamor-Danek, Memphis, TN). Alternatively, it can be estimated by placing a rod template on the skin at the back of the patient (Frontier system, De-Puy, AcroMed, Raynham, MA). The cephalic and caudal ends of the rod are determined via fluoroscopic localization of the respective ends of the instrumented spinal levels on the template. The use of an appropriate rod length is encouraged. An excessive length at the distal end of the rod-screw complex is to be avoided or otherwise it may cause diaphragmatic impingement and irritation. The rod is normally inserted using the cantilever method (Figure 12) without contouring although it may be contoured if required.

The cantilever method of scoliotic reduction puts the most cephalic placed screws at highest risk of plowing and pullout. ${ }^{[6]}$ To avoid this, the rod can be engaged to the screws in a cephalocaudal direction. Bicortical screw purchase and the additional placement of staple-screw complex can further enhance screw stability in the vertebral body. The rod-screw construct is secured by insertion of setscrews. Intervertebral compression is performed

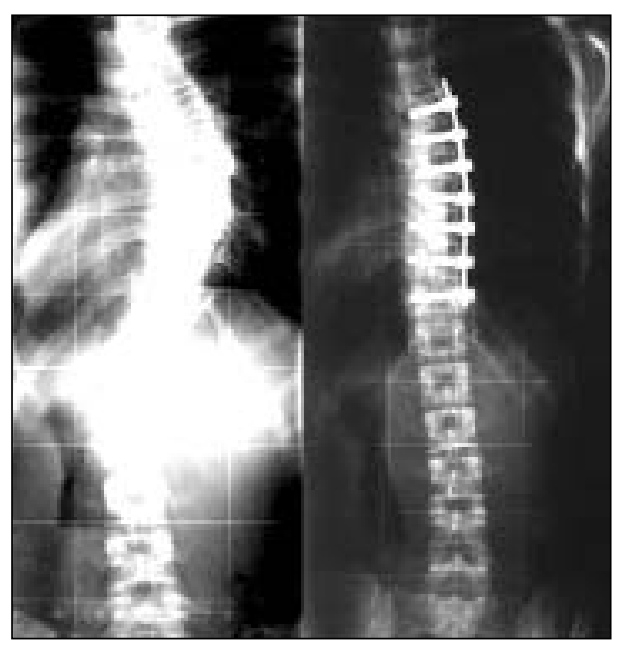

Figure 13: Shows the preoperative (left) and postoperative (right) PA radiographic images of the patient using Eclipse instrumentation. in a cephalo-caudal direction. Excessive compression and over correction can be avoided by ensuring a parallel end plates configuration of adjacent vertebrae under fluoroscopic control during intervertebral compression.

Following the tightening of all screws, the entire construct is visualized in an $\mathrm{AP}$ and lateral fluoroscopic views. The parietal pleural can be closed or left open after the procedure. Newton et $\mathrm{al}^{[26,27]}$ advocates the closure of the pleura with a running 2-0 absorbable suture, using the Endositch device (U.S. Surgical corp.). Closure of the parietal pleural may reduce postoperative chest tube drainage and help to retain bone graft in the post discectomy disc space. Other surgeons ${ }^{[1,7,40]}$ prefer to leave the pleura open. A fibrous layer develops over the implants by several weeks after the surgery. ${ }^{[7,18]}$

A chest tube for postoperative surgical drainage is inserted through a caudal portal. We prefer to use the supplementary $5 \mathrm{~mm}$ retraction port instead of one of the working ports for chest tube placement to avoid excessive post-operative fluid leakage around the chest tube. Depending on the surgical protocols the patient may be extubated ${ }^{[18,27]}$ or kept intubated with an exchange of a single-lumen endotreacheal tube during the first postoperative night in the intensive care unit (ICU) to ensure a smooth alveolar reinflation. ${ }^{[40]}$ Routine suction of the inflated lung is recommended to remove mucous plugs, which cause significant respiratory distress. ${ }^{[1,30]}$

A supportive front-back shell TLSO is used for three months. Interbody fusion is usually seen at six months and a good spinal fusion should expect at one year after the operation.

\section{Complications}

McAfee et $\mathrm{al}^{[20]}$ reported the most common complications encountered in VATS in spinal disorders were intercostal neuralgia $(7.7 \%)$ and symptomatic atelectasis $(6.4 \%)$. Others VATS related complications that have been reported are: excessive (>2000cc) intraoperative blood loss $(2.5-5.5 \%),{ }^{[12,20]}$ pneumonia (1$3 \%),{ }^{[2,12,26]}$ wound infections $(1-3 \%),{ }^{[12,26]}$ chylothorax $(1 \%),{ }^{[2,12,26]}$

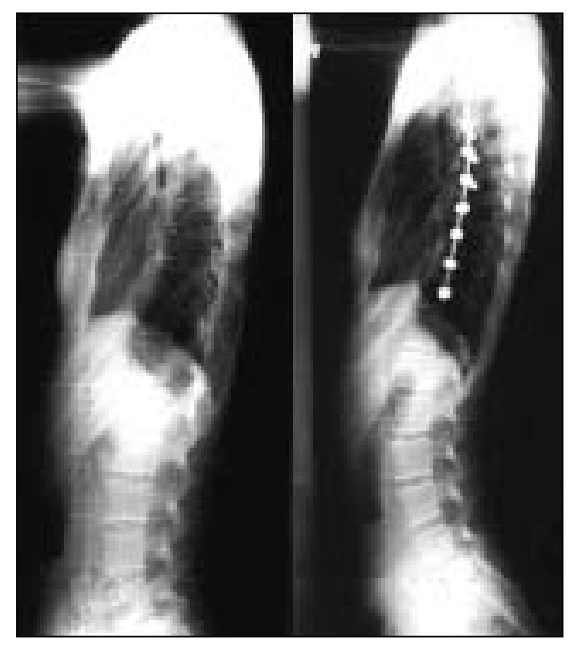

Figure 14: Shows the preoperative (left) and postoperative (right) lateral radiographic images of the patient using Eclipse instrumentation 
hemidiaphragm ${ }^{[20]}$ and pericardial penetration, ${ }^{[12]}$ tension pneumothorax, ${ }^{[2,33]}$ long thoracic nerve injury, ${ }^{[40]}$ pulmonary embolism $^{[2]}$ and a case of simultaneous bilateral pneumothoraces, pneumomediastinum, pneumoretroperitoneum with subcutaneous emphysema following after a double lumen endotreacheal intubation. ${ }^{[34]}$

Obtaining an adequate emergency vascular control in thoracoscopic surgery is potentially difficult. Sucato et al has highlighted the possibility of injury to the thoracic aorta from vertebral body screws at the apex of the scoliotic curve. ${ }^{[37]}$ This is because the thoracic aorta often lies on the left side of the vertebral body in scoliosis instead of the more anterior position in normal patients, and inappropriately long screws inserted from the right side could penetrate the thoracic aorta. For the same reason, it is not advisable to instrument left sided curves from the convex side. In general, thoracotomy instrumentation should always be available during thoracoscopic procedures to cater for such surgical emergencies.

\section{VATS results in various spine conditions Thoracic disc disease}

Rosenthal and Dickman reported the results of 55 consecutive patients undergoing VATS discectomy ${ }^{[32]} 60 \%$ of the myelopathic patients recovered neurologically, whereas $79 \%$ of the radiculopathic patients recovered completely. When compared the VATS results to their patient treated by costotransversectomy or thoracotomy, they found VATS was associated with $50 \%$ less blood loss and an hour less operative time. Anand and Regan ${ }^{[3]}$ reported their results of 100 consecutive cases of thoracic disease treated by VATS. They classified the disease according to the symptoms: Grade 1 (pure axial), Grade 2 (pure radicular), Grade $3 \mathrm{~A}$ (axial and thoracic radicular), Grade 3B (axial with lower leg pain), Grade 4 (myelopathic) and Grade 5 (paralytic). The mean operative time was 173 minutes and average intra-operative blood loss was $259 \mathrm{ml}$. A significant improvement in Oswestry score was noted in Grade 4; followed by Grade 3A, 3B and Grade 1 patients. An overall subjective patient satisfactory rate was $84 \%$ and objective long-term clinical success was obtained in $70 \%$ of patients at 2 years. It was interesting to note that in Grade 2 patients, those with pure thoracic radicular pain, their radicular symptom did improve initially after the operation. However most of these patients complained of axial pain in the subsequent follow up assessment.

\section{Spine fracture}

Dickman et al. reported a comparable outcome in fracture management between VAT- vertebrectomy and open thoracotomy group. ${ }^{[9]}$ Although both the operative time and intra-operative blood loss were comparable between the two groups, there was a significant reduction in narcotic use; ICU and hospital stay in VATS group. To further simplify the technique; Huang et al described the use of an extended manipulating VATS channel. An extended (or working) portal of 5 to $6 \mathrm{~cm}$ in length was placed at the injured level or behind the posterior axillary line at T9-10 space to allow an easy fracture reconstruction. ${ }^{[11]}$

\section{Spine tumor}

Many authors had described the use of VATS in management of primary and metastatic spinal tumors..$^{[8,12,15,21,31]}$ Konno et al reported the use of a combined hemi-laminectomy with medial facetectomy via a standard posterior approach and thoracoscopic resection for the management of 5 dumbbell-type thoracic cord tumors. No instrumentation was used. All patients regained their ability to walk. There was no recurrence of tumor and spinal instability at 3 years after the operation. However the use of VATS in these high-risk patients has to be exercised with caution. In a series of 41 patients with metastatic tumor decompressed by VATS, there were $2(5 \%)$ perioperative deaths and both were related to respiratory complications. ${ }^{[12]}$

\section{Vertebral osteomyelitis}

The use of VATS to obtain tissue confirmation for a faster and more reliable diagnosis of thoracic spinal tuberculosis has been reported. ${ }^{[10]}$ Endoscopic approach to the treatment of thoracic vertebral osteomyelitis may reduce the surgical morbidity that is otherwise untolerated in these sick patients. ${ }^{[12,13,20,23]}$

Huang et al demonstrated the effectiveness and safety of using VATS in the management of tuberculous spondylitis in 10 patients. ${ }^{[13]} 9$ of 10 patients had neurological deficit. A triangulated 3 portal placement, using the extended manipulating channel method was performed. Autogenous tricortical iliac graft was used in most patients for post-debridement anterior spinal column reconstruction. No posterior spinal stabilization was done. Mean operating time was 2.9 hours and mean intraoperative blood loss was $485 \mathrm{ml}$. Intrathoracic adhesion was noted in $40 \%$ of the patients and this resulted in one conversion into open surgery. The average patient neurologic recovery was 1.1 Frankel grades. At 2 years after surgery, there was no recurrence of infection but increased kyphotic deformity in 2 patients secondary to rib graft subsidence. Although, no anterior instrumentation was used in this series, the save usage of such implant had been reported. ${ }^{[5]}$

Muckley et al reported the management of 3 elderly patients with pyogenic vertebral osteomyelitis and epidural abscess by VATI. ${ }^{[23]}$ Radical debridement, ipsilateral pedicle resection of the pathological vertebrae, lead to direct visualization of the dural sac and spinal canal decompression were performed. Interbody fusion and kyphotic deformity correction were achieved with an expandable titanium interbody fusion cage containing autogenous bone graft and gentamycin impregnated collagen sponge. The construct was further stabilized with an anterior fixation system. No brace was used postoperatively. There was no recurrence of infection and no loss of postoperative kyphotic correction at 2 year. Operative time and blood lost were comparable to open techniques.

\section{Scoliosis correction}

Thoracoscopic surgery for scoliosis is presented used in one of two forms. Anterior spinal release with fusion ${ }^{[1,2,7,14,18,24-26,36,38]}$ or 
anterior spinal release with fusion and anterior instrumentation. ${ }^{[27,30,40]}$ Anterior spinal release is used for severe or rigid curves or in young patients where there is a need to achieve anterior and posterior spinal fusion. Thoracoscopic anterior release and fusion is done as the first stage of a two stage procedure. The second stage is conventional posterior fusion and instrumentation.

Arlet published a meta-analysis of anterior thoracoscopic spine release in scoliotic deformity surgery. ${ }^{(4)}$ He found an average of 4 to 7 discs was excised with an operative time varied between 150240 minutes. The average Cobb angle of the structural curve was $65^{\circ}$. The percentage of curve correction was 55-63\% after VATS and posterior spine fusion. The average hospital stay was 9 days. The total complication reported was $18 \%$ and most were pulmonary complications noted in patients with neuromuscular deformity. In one series, the author noted a $28 \%$ cost increased in VATS when compared to standard thoracotomy. The conversion rate from VATS into thoracotomy found in series with over 100 cases was from 0 to $3 \% .^{[2,26]}$

We started performing thoracoscopic fusion and anterior instrumentation for patients with adolescent idiopathic scoliosis in 2000. In 2003, we reviewed our experience with this technique and compared the results thoracoscopic instrumented fusion with those patients who had conventional posterior instrumented fusion. ${ }^{[40]}$ Consecutive patients with adolescent idiopathic scoliosis who had selective thoracic fusion with either technique and who had a minimum follow-up of 2 years were included in this study. Posterior instrumentation in this series was all-hook systems. We found no difference in outcome between the two groups with regard to post-operative Cobb angle, thoracic kyphosis and lumbar lordosis at different time points in 2 years. Cobb angle correction was $66 \%, 62 \%$, and $62 \%$ at one week, 6 months, and 2 years post-operatively respectively for the thoracoscopic group; and $75 \%, 70 \%$, and $65 \%$ at one week, 6 months, and 2 years postoperatively respectively for posterior instrumentation. There was a trend towards better correction with posterior instrumentation, but the differences between the two groups were not statistically significant. Operative time was significantly longer than for conventional posterior instrumented fusion ( 7 hours versus 4 hours); blood loss was less; and ICU stay was longer with the thoracoscopic method compared to posterior instrumentation. However, the thoracoscopic method saved on the average 3.5 fusion levels compared to posterior fusion.

Newton et al, in a report to the Scoliosis Research Society in 2002 compared anterior thoracoscopic instrumented fusion to anterior open and posterior instrumented fusion in a cohort of patients from a number of surgeons. He found similar outcomes in all three approaches. There was a trend towards better correction in the posterior instrumented group, but the differences were not statistically significant. The thoracoscopic group had longer operating times. In another report on patients from the same study group, 38 thoracoscopic instrumentation cases with six months' follow-up were compared to 68 anterior open instrumentation cases. ${ }^{[25]}$ The average Cobb angle correction averaged $60 \%$ and was similar for both groups. Reduction in forced vital capacity was significantly greater in the open group when compared to the thoracoscopic group. Another advantage of thoracoscopic instrumentation over open thoracotomy is less operative post-operative pain. ${ }^{(29)}$ In this report, Picetti et al also reported an improvement in curve correction in their last 10 cases, which was attributed to overcoming the learning curve.

In a retrospective review of our learning curve, we looked at 50 consecutive skeletal immature patients with adolescent idiopathic scoliosis (AIS) who were treated by VAT-I. The minimum followed up was 6 months ( $6-54$ months). The average age of the patient at surgery is $14 \pm 2.1$ years old. The indications for surgery included King type 3, type 2 and Lenke type 1 curves. The average preoperative curves size was $48.4 \pm 8.8^{\circ}$. The average pre-operative right bending radiograph showed a curve reduction to $29.9 \pm$ $11.7^{\circ}$. The average pre-operative kyphosis is $20.4 \pm 12.3^{\circ}$.

All the patients were operated on by a single surgeon (WHK). Spinal fusion levels were selected to fuse from end vertebra to end vertebra. A lateral decubitus patient position and a double-lumen endotreacheal tube were used during anesthesia. An all posterolateral approach with initial fluoroscopic portal localization technique was used. This was subsequently replaced by the standardized four portal approach without the need of any fluoroscopic localization. Discectomies were performed successfully without the rib head resection. Anterior longitudinal ligament was partially released. No intra-operative radiograph was required for spinal level confirmation in the later part of this surgical series. The spinal level was gauged by identifying the previous described anatomical landmarks. The placement of the screws was subsequently performed by direct screw placement technique with an awl. A straight single rod is applied to a single screw construct. The rod is captured in a cephalo-caudal direction. The scoliotic deformity is corrected by cantilevered method. Autogenous rib grafts were used for spinal fusion. No pleura were closed and a postoperative $20 \mathrm{FG}$ chest-tube was inserted via the $5.5 \mathrm{~mm}$ diameter portal in the $8^{\text {th }}$ intercostal space (Figure 1). Patients were kept intubated with a single lumen endotreacheal tube during the first postoperative night in the ICU unit. A TLSO was prescribed to the patient for three months postoperatively. The patient was excused from $\mathrm{PE}$ for one year.

The average operative time used was ranged from 302 to 372 minutes. The blood lost range from 175 to $266 \mathrm{ml}$. The average ICU stays were 1.8 to 3.0 days. The average hospital stays were 6.9 to 7.5 days. The average Cobb angle obtained in a coronal plane was $15^{\circ}$. No major complication was noted except one patient with lobar collapse and the other patient with a long thoracic nerve paralysis. In a sub-group analysis where the initial 25 patients' results were compared to that of the latter 25 patients', there was a statistically significant better scoliosis correction noted in the last 25 cases. In addition, a shorter ICU, hospital stay and operative time were noted. The shorter operative time was accredited to a reduced operating theatre set-up time, the avoidance of the pre-operative fluoroscopic portal locations, faster discectomies and an improvement in anesthetist learning curve. 
Curve correction was also better. In the first 25 patients, Cobb angle correction was $67 \%$ and $60 \%$ at one week and 6 months post-operatively respectively; while for the next 25 patients, Cobb angle correction was $80 \%$ and $76 \%$ for the same time periods (Figures 13 and 14). An improved scoliotic correction might be of the result to a more posterior screw placement and a more effective sequential intervertebral compression during scoliosis curve correction. Using locally weighted regression fit, the learning curve for shorter operating time and ICU stay was 30 cases; and 20 cases was required for better Cobb angle correction.

\section{Summary}

Video assisted thoracoscopic surgery and instrumentation is a safe and effective method in treating a variety of spinal conditions. It is especially useful in correcting adolescent idiopathic scoliosis deformity. It gives comparable results in terms of Cobb angle correction and sagittal alignment to open anterior or posterior instrumented fusion. The advantages of the thoracoscopic approach are the avoidance of double thoracotomies in a standard open anterior approach, as well as the long posterior incision and paraspinal muscle dissection in a posterior approach. Compared to open anterior, there is also less pain and less interference with lung function. The 'keyhole' incisions are expected to be cosmetically more pleasing that the long thoracotomy or long posterior midline incision. The disadvantages of the thoracoscopic approach are the steep learning curve, keeping up with evolving surgical technique and instrumentation, and higher costs. It is also difficult at the present time to treat large and rigid curves, as well as to approach the thoracic spine above T4 or obtaining emergency vascular control compared to an open approach. However, when one gets over the steep leaning curve, the results of VATS are often very pleasing to both the patient and the surgeon.

\section{References}

1. Al-Sayyad M, Crawford A, Wolf R. Early experiences with video-assisted thoracoscopic surgery: Our first 70 cases. Spine 2004;29:1945-51.

2. Al-Sayyad M, Crawford A, Wolf R. Video-assisted thoracoscopic surgery. The Cincinnati experience. CORR 2005;434:61-70.

3. Anand N, Regan J.J. Video-assisted thoracoscopic surgery for thoracic dise disease:classification and outcome study of 100 consecutive cases with a 2-year minimum follow-up period. Spine 2002;27:871-9.

4. Arlet V. Anterior thoracoscopic spine release in deformity surgery: a meta-analysis and review. Eur Spine J 2000;9:S17-23.

5. Benli IT, Alanay A, Akalin S, Kis M, Acaroglu E, Ates B, et al. Comparison of anterior instrumentation systems and the results of minimum 5 year follow-up in the treatment of Tuberculosis spondylitis. Kobe J Med Sci 2004;50:167-80.

6. Betz R, Lenke L, Harms J, Lowe T, Clements D. Anterior spinal fusion and instrumentation for adolescent scoliosis. Semin Spine Surg 1998;10:88-94.

7. Crawford AH, Wall E.J, Wolf R. Vedio- assisted thoracoscopy. Orthop clin North Am 1999;30:367-85.

8. Citow JS, MacDonald RL, Ferguson MK. Combined laminectomy and thoracoscopic resection of a dumbbell neurofibroma. Technical case report. Neurosurgery 1999;45:1263-6.

9. Dickman CA, Rosenthal D, Karahalios DG, Paramore CG, Mican CA, Apostolides PJ, et al. Thoracic vertebrectomy and reconstruction using a microsurgical thoracoscopic approach. Neurosurgery 1996;38:279-93.

10. Dusmet M, Halkic N, Corpataux J. Video-assisted thoracie surgery disgnosis of thoracic spinal tuberculosis. Chest 1999;116:1471-2.

11. Huang T.J, Hsu RW, Liu HP, Hsu KY, Liao YS, Shih HN, et al. Video-assisted thoracoscopic treatment of spinal lesions in the thoracolumbar junction. Surg Endose 1997;11:1189-93.

12. Huang TJ, Hsu RW, Sum CW, Liu HP. Complications in thoracoseopic spinal surgery A study of 90 consecutive patients. Surg Endose 1999;13:346-50.

13. Huang T.J, Hsu R, Chen SH, Liu HP. Video-assisted thoracoscopic surgery in managing Tuberculous spondylitis. Clin Orthop Relat Res 2000;379:143-53.

14. King AG, Mills TE, Leo WA Jr, Chutkan NG, Revels TS. Video-assisted thoracoscopic surgery in the prone position. Spine 2000;25:2403-6.

15. Konno S, Yabuki S, Kinoshita T, and Kikuchi S. Combined laminectomy and thoracoscopic resection of dumbbell-type thoracic cord tumor. Spine 2001;26:E130-4.

16. Kuklo TR, Lenke LG. Thoracoscopic spine surgery:Current indications and techniques. Orthopaedic Nursing 2000;19:15-22.

17 Lenke LG, Betz RR, Harms J, Bridwell KH, Clements DH, Lowe TG, et al. Adolescent idiopathic scoliosis: A new classification to determine extent of spinal of spinal arthrodesis. J Bone Joint Surgery Am 2001;83:1169-81.

18. Lenke LG. Anterior endoscopic discectomy and fusion for adolescent idiopathic scoliosis. Spine 2003;28:S36-43.

19. Lieberman IH, Salo PT, Orr RD, Kraetchmen B. Prone position endoscopic transthoracic release with simultaneous posterior instrumentation for spinal deformity: A description of the technique. Spine 2000;25:2251-7.

20. McAfee PC, Regan JJ, Zdeblick T, Zukerman J, Picetti GD, Heim S, et al. The incidence of complications in endoscopic anterior thoracolumbar spinal reconstructive surgery. A prospective multicenter study comprising the first 100 consecutive cases. Spine 1995;20:1624-32.

21. MeLain R, Lieberman H. Controversy. Endoscopic approaches to metastatic thoracic disease. Spine 2000;25:1855-8

22. Mehlman CT, Crawford AH, Wolf RK. Video-assisted thoracoscopic surgery (VATS). Endoscopic thoracoplasty technique. Spine 1997;22:2178-82.

23. Muckley T, Schutz T, Schmidt M, Potulski M, Buhren V, Beisse R. The role of thoracoscopic spinal surgery in the management of pyogenic vertebral osteomyelitis. Spine 2004;29:E227-33.

24. Newton P, Shae KG, Granlund KF. Defining the pediatric spinal thoracoscopy learning curve: Sixty-five consecutive cases. Spine 2000;25:1028-35.

25. Newton PO, Marks M, Faro F, Betz R, Clements D, Haher T, et al. Use of videoassisted thoracoscopic surgery to reduce perioperative morbidity in scoliosis surgery. Spine 2003;28:S249-54.

26. Newton P, White K, Faro F, Gaynor T. The success of thoracoscopic anterior fusion in a consecutive series of 112 pediatric spinal deformity cases. Spine 2005;30:392-8.

27. Newton P, Parent S, Marks M, Pawelek J. Prospective evaluation of 50 consecutive scoliosis patient surgically treated with thoracoscopic anterior instrumentation. Spine 2005;30:S100-9.

28. Orchowski J, Bridwell K, Lenke L. Neurological deficit from a purely vascular etiology after unilateral vessel ligation during anterior thoracolumbar fusion of the spine. Spine 2005;30:406-10.

29. Picetti G, Ertl J, Bueff U. Endoscopic instrumentation, correction, and fusion of idiopathic scoliosis. Spine 2001;1:190-7.

30. Picetti G, Pang D. Thoracoscopic techniques for the treatment of scoliosis. Childs Nerv Syst 2004;20:802-10.

31. Rosenthal D. Endoscopic internal fixation of the thoracic spine. In: Regan J.J, McAfee PJ, Mack MJ editors. Atl Endoscop Spine Surg 1995. p. 333.

32. Rosenthal D, Dickman CA. Thoracoscopic microsurgical excision of herniated thoracic dises. J Neurosurgery 1998;89:224-35.

33. Roush T, Crawford AH, Berlin RE, Wolf RK. Tension pneumothorax as a complication of video- assisted thoracoscopic surgery for anterior correction of idiopathic scoliosis in an adolescent female. Spine 2001;26:448-50.

34. Sucato D, Magda G. Case report. Bilateral pneumothoraces, pneumomediastinum, pneumoperitoneum, pneumoretroperitoneum, and subcutansous emphysema following intubation with a double -lumen endotracheal tube for thoracoscopic anterior spinal releases and fusion in a patient with idiopathic scoliosis. J Spinal Disord Tech 2002;15:133-8.

35. Sucato DJ, Welch RD, Pierce B, Zhang H, Haideri N, Bronson D. Thoracoscopic discectomy and fusion in an animal model: safe and effective when segmental blood vessels are spared. Spine 2002;15:880-6.

36. Sucato D, Elerson E. A comparison between the prone and lateral position for performing a thoracoscopic anterior release and fusion for pediatric spinal deformity. Spine 2003;28:2176-80.

37. Sucato D, Kassab F, Dempsey M. Analysis of screw placement relative to the aorta and spinal canal following anterior instrumentation for thoracic idiopathic scoliosis. Spine 2004;29:554-9

38. Waisman M, Saute M. Thoracoscopic spine release before posterior instrumentation in scoliosis. Clin Orthop 1997;336:130-6.

39. Winter RB, Lonstein JE, Denis F, Leonard AS, Garamella JJ. Paraplegia resulting from vessel ligation. Spine 1996;21:1232-3.

40. Wong HK, Hee HT, Yu Z, Wong D. Results of thoracoscopic instrumented fusion versus conventional posterior instrumented fusion in adolescent idiopathic scoliosis undergoing selective thoracic fusion. Spine 2004;29:2031-8. 